\title{
Distracting spontaneous refractory hypoglycaemia
}

Seong K Cheah ${ }^{1}$, Roby Rajan ${ }^{1}$, Abraham Mathews ${ }^{1}$, John Grant ${ }^{2}$, Singhan M Krishnan ${ }^{1}$, Anitha Mathews ${ }^{1}$, Shyam S Seshadri ${ }^{1}$

${ }_{1}^{1}$ Department of Endocrinology and Diabetes, Hinchingbrooke Hospital, North West Anglia Foundation Trust, UK

${ }^{2}$ Department of Pathology, Addenbrooke Hospital, UK

\section{BACKGROUND}

Spontaneous hypoglycaemia in non-diabetic individuals warrants investigation. Among the usual suspects, insulinoma is a more well described entity compared to the less commonly reported counterpart, proinsulin secreting neuroendocrine tumour. A series reported proinsulinoma has a 2:1 female preponderance with mean age of diagnosis at 56 years old (1). The lesion usually lies, unsurprisingly, at the body and tail of the pancreas, which signposts to the diagnosis (1-3). However, other concomitant malignancy or extra-pancreatic location may cloud the diagnosis. We herein report a case of such diagnostic conundrum.

\section{CASE HISTORY}

A 79 years old frail lady with history of dementia and hypertension presented with refractory hypoglycaemia over a period of 3 months requiring multiple admissions. During her most recent admission, intermittent octreotide and glucocorticoid were tried but she still required continuous dextrose infusion to maintain euglycaemia.

The severe spontaneous hypoglycaemia in this non-diabetic lady, warranted a series of investigations.

TFT excluded thyroid dysfunction

Short synacthen test excluded hypoadrenalism

The anterior pituitary profile including prolactin, $\mathrm{LH}$, and FSH were all normal.

Two separate samples of IGF-I was $8 \mathrm{nmol} / \mathrm{L}$ and $6.6 \mathrm{nmol} / \mathrm{L}(10-25 \mathrm{nmol} / \mathrm{L})$.

CT scan with contrast of the abdomen and pelvis revealed a heterogeneously enhancing mass $(6.6 \mathrm{~cm})$ arising from the lower pole of the left kidney consistent with renal cell carcinoma. Concomitantly there were extensive peripherally enhancing heterogeneous mass lesions in the liver, the largest measuring at $12 \mathrm{~cm}$. The pancreas was normal.

During the event of hypoglycaemia (blood glucose $1.6 \mathrm{mmol} / \mathrm{L}$ ), the blood tests revealed:

IGF-II:IGF-I ratio less than 10, which was inconsistent with non-islet cell tumour induced hypoglycaemia (NICTH).

Inappropriate elevation of C-peptide 4210pmol/L (174-960pmol/L)

Inappropriate elevation of Proinsulin $>200(0-7 \mathrm{pmol} / \mathrm{L})$

Suppressed Insulin at $12 \mathrm{pmol} / \mathrm{L}(0-180)$

In view of multiple comorbidities, a palliative approach was taken. The post-mortem confirmed a clear cell renal carcinoma of the left kidney. Unexpectedly, the morphology and immunoprofile of the liver metastases were consistent with proinsulin secreting neuroendocrine tumour.

The immunostaining showed focal strong insulin immunoreactivity, as well as widespread CD56, synaptophysin, and chromogranin A, with negative staining for RCC.

\section{CONCLUSION AND LEARNING POINTS}

Proinsulinoma is a rare condition and can be masked by concomitant metastatic malignancy. Multiple hepatic metastases is a well-known cause of spontaneous hypoglycaemia. However, in severe intractable hypoglycaemia, coexistence of insulin secreting tumour needs to be considered to avoid missing them.
IMAGING
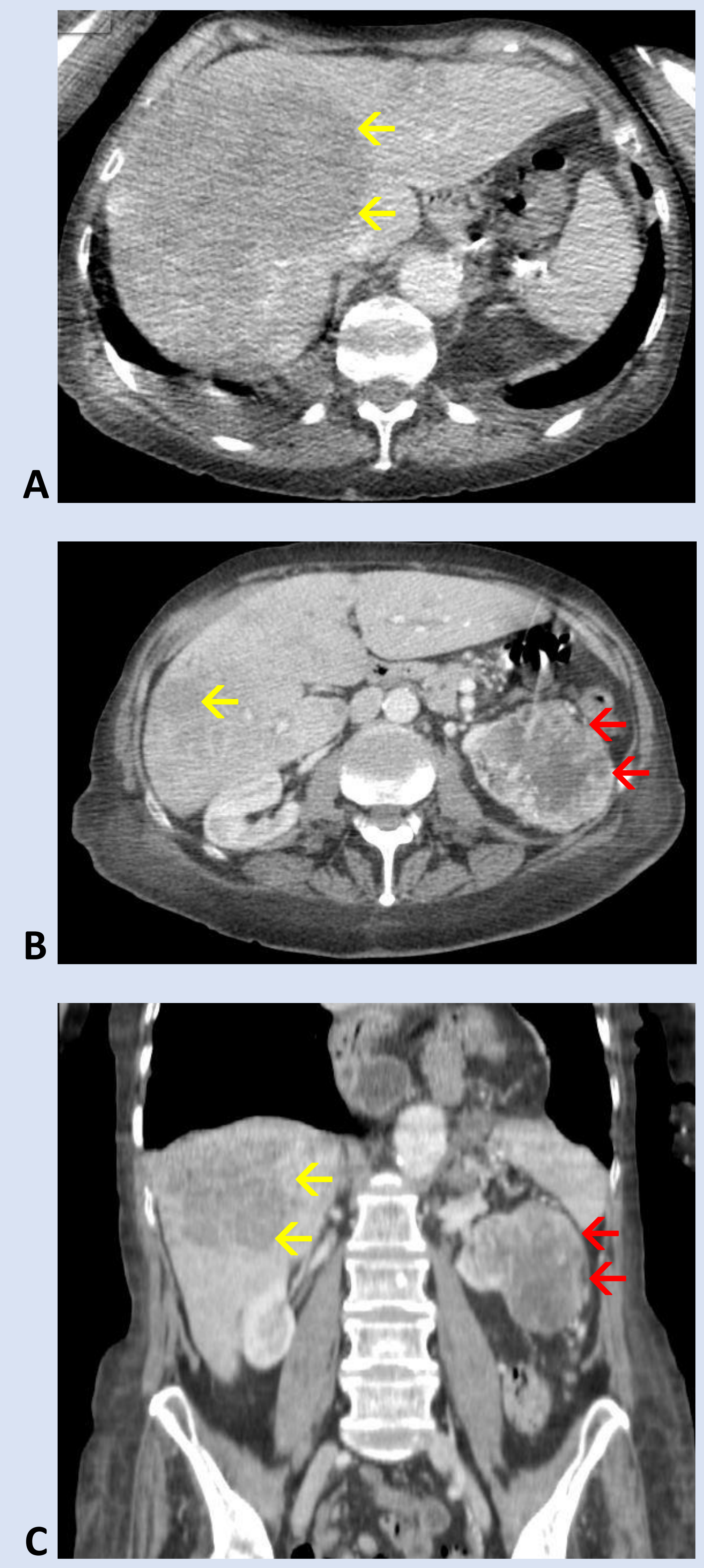

Fig 1. CT abdomen and pelvis of transverse $(A, B)$ and coronal (B) view demonstrating the renal (red arrows) and liver lesions (yellow arrows)
Fig 2. Liver tumour A: H\&E staining $(x 10)$

B: Synaptophysin staining $(x 10)$

C: Insulin immunoreactivity (x20)

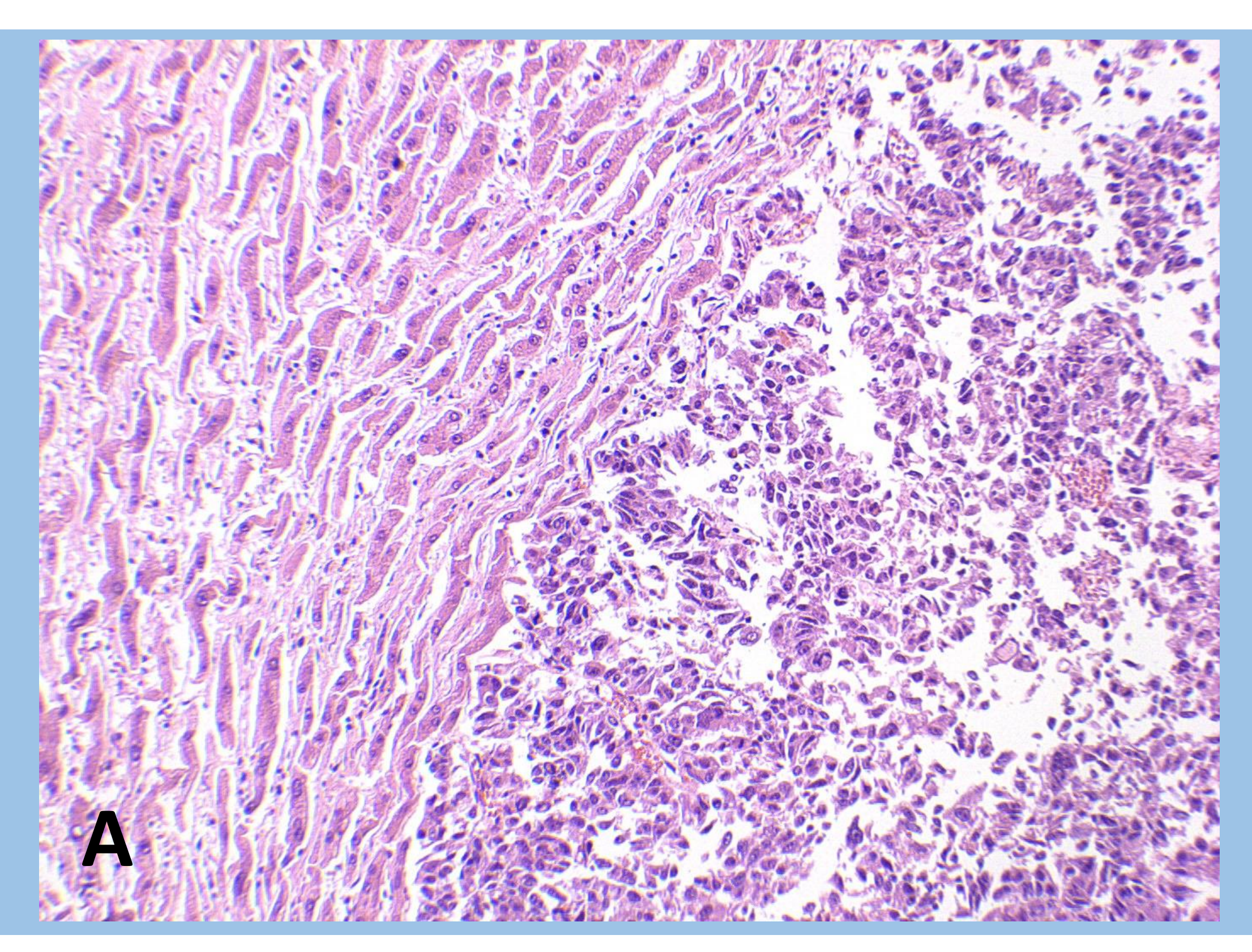

B
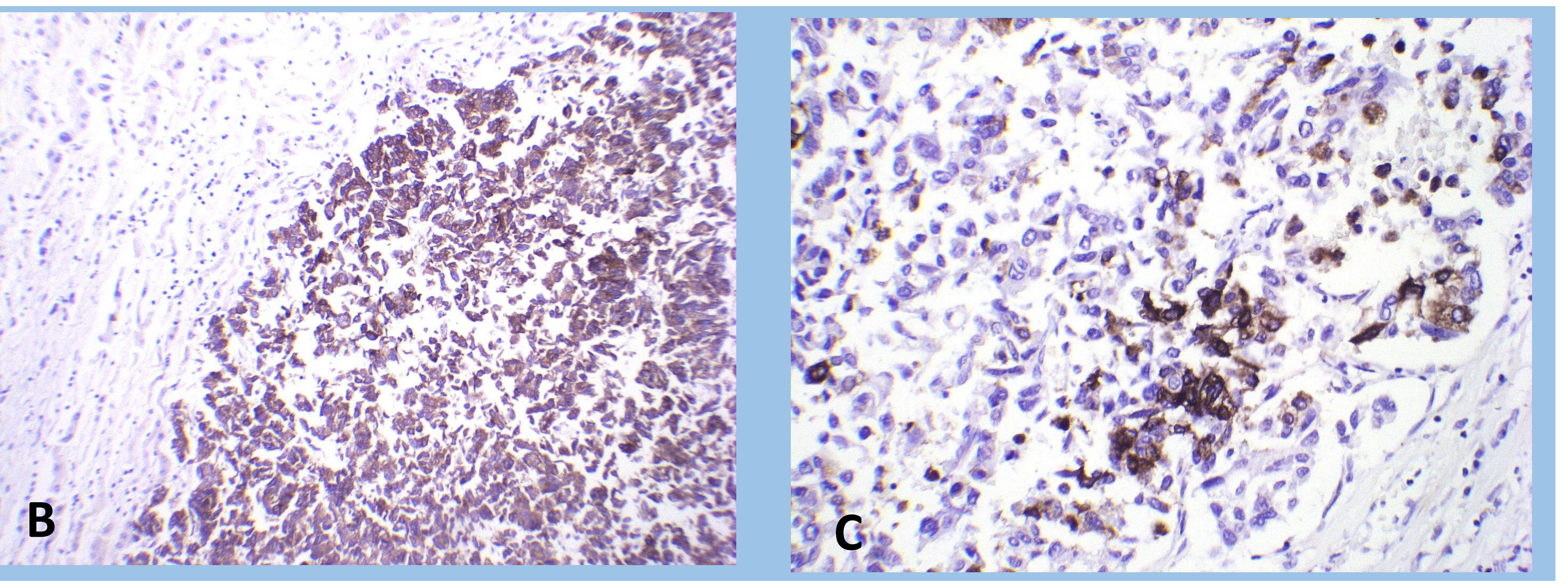

Fig 3. Kidney tumour

A: H\&E staining $(x 10)$

$B$ : Synaptophysin staining $(x 10)$

C: Insulin immunoreactivity (x10)

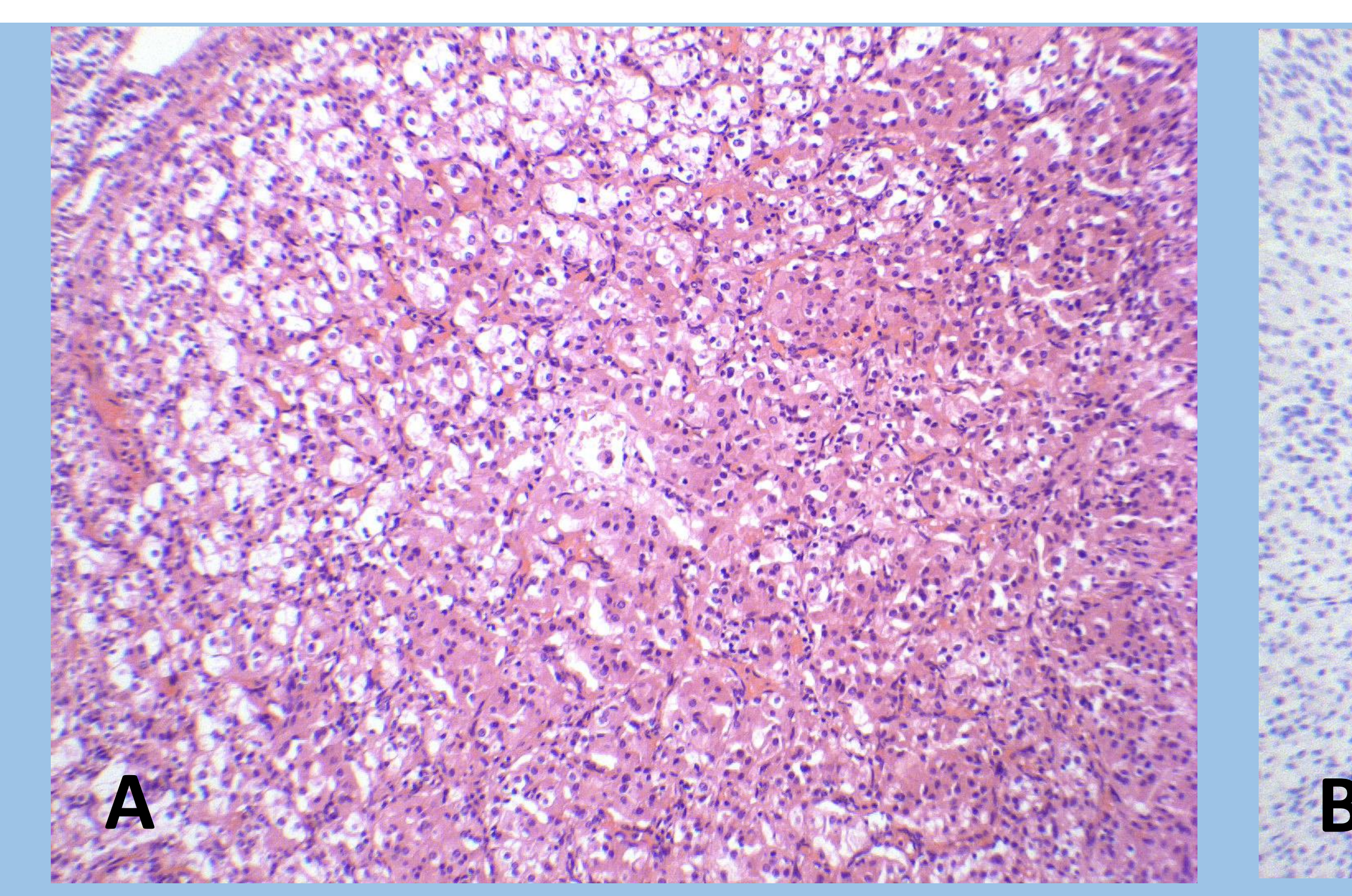

\section{REFERENCE}

1. Murtha TD, Lupsa BC, Majumdar S, Jain D, Salem RR. A Systematic Review of Proinsulin-Secreting Pancreatic Neuroendocrine Tumors. J Gastrointest Surg. 2017 Aug 16;21(8):1335-41.

2. Fadini GP, Maran A, Valerio A, Meduri F, Pelizzo M, Miotto D, et al. Hypoglycemic Syndrome in a Patient with Proinsulin-Only Secreting Pancreatic Adenoma (Proinsulinoma). Case Rep Med. 2011;2011:1-5.

3. Pérez-Pevida B, Idoate MÁ, Fernández-Landázuri S, Varo N, Escalada J. Hypoglycemic Syndrome without Hyperinsulinemia. A Diagnostic Challenge. Endocr Pathol. 2016 Mar 22;27(1):50-4. 